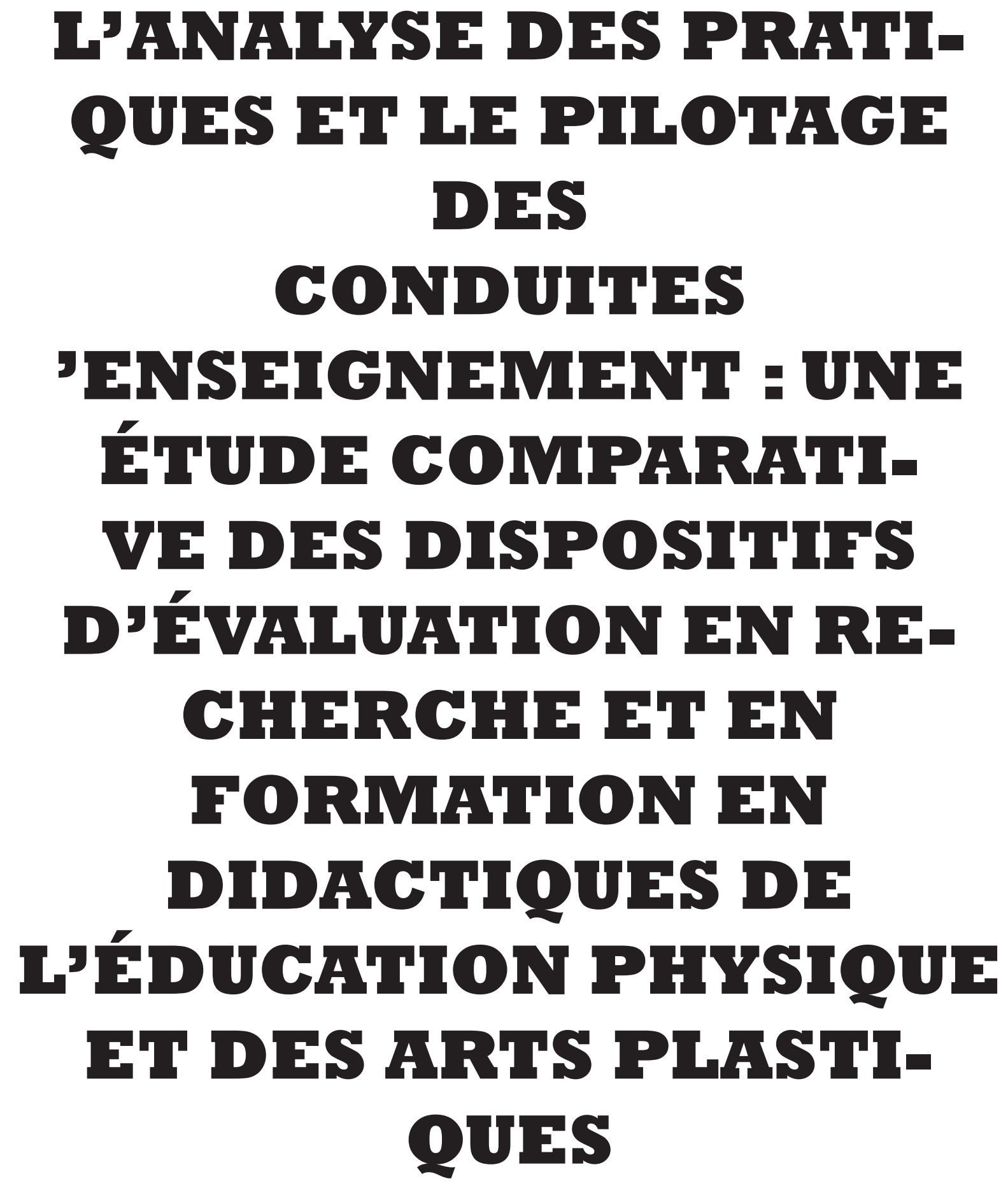




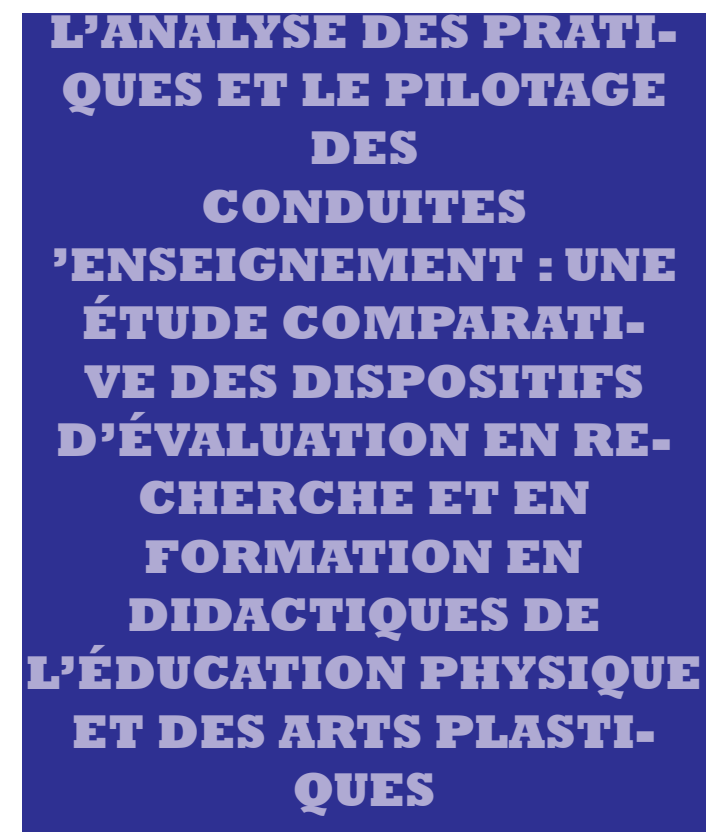

Résumé. L'évaluation constitue une véritable problématique dans le cadre de l'analyse des pratiques visant la formation aux gestes professionnels de conduite des séquences d'enseignement/apprentissage dans le champ des didactiques des arts plastiques et/ou de l'éducation physique. Elle tient autant des conceptions générales du rôle de l'école dans le développement des enfants que des aspects pragmatiques liés aux significations et à la " manière » de conduire les activités d'enseignement/apprentissage.

Notre travail analyse un dispositif de recherche sur les rapports entre les éléments d'intelligibilité de la pratique enseignante qui émergent à travers un dispositif d' "analyse et d'évaluation des leçons ", d'une part, et leur mobilisation (ou leur non mobilisation) dans la conduite des leçons par les enseignants titulaires (généralistes) et des enseignants spécialistes amenés à co-gérer une séquence d'enseignement, d'autre part.

Les données sont issues de deux mises en œuvre de ce dispositif auprès de deux situations contrastées :

- Dispositif appliqué à la formation initiale des enseignants dans le cadre de la gestion en co-responsabilité (par des stagiaires) d'une séquence didactique en arts plastiques ;

- Dispositif appliqué à une recherche sur la gestion en co-responsabilité (enseignants généraliste et spécialiste) d'une séquence d'enseignement en éducation physique.

Adrián Cordoba - René Rickenmann

Université de Genève 
Du point de vue méthodologique, l'analyse est centrée sur la transcription écrite des leçons d'une séquence d'enseignement gérée en co-responsabilité par les deux types d'enseignants, ayant été vidéoscopées. Nos catégories d'analyse sont issues du modèle descriptif de l'action enseignante proposé par Mercier, Sensevy \& Schubauer-Leoni (2000), ainsi que des concepts de l'action enseignante proposés dans la théorie des situations didactiques (Brousseau, 1996). Du point de vue méthodologique, le dispositif s'inspire des propositions sur l'autoconfrontation croisée dans le champ de l'analyse du travail (par exemple, Clot \& Faïta, 2000), mais avec les adaptations mises en oeuvre par plusieurs chercheurs dans le champ de la formation initiale et continue des enseignants (Goigoux, sous presse ; Lenoir \& ali, 2002).

Mots-clés. autoconfrontation, gestes professionnels, évaluation formative

\section{EL ANÁLISIS DE LAS PRÁCTICAS Y EL PILOTAGE DE LAS CONDUCTAS DE ENSEÑANZA: UN ESTUDIO COMPARATIVO DE LOS DISPOSITIVOS DE EVALUACIÓN EN INVESTIGA- CIÓN Y EN LA FORMACIÓN EN DIDACTICAS DE LA EDUCACIÓN FÍSICA Y DE LAS ARTES PLÁSTICAS}

Resumen. La evaluación constituye una verdadera problemática en el marco de los análisis sobre los dispositivos de formación acerca de los gestos profesionales de las unidades de enseńanza-aprendizaje en las didácticas de la educación física y/o de las artes plásticas y visuales. Esta depende, tanto de las concepciones generales sobre el rol de la escuela en el desarrollo del nińo, como de los aspectos pragmáticos relacionados con las significaciones y con las "maneras" de manejar las actividades en el aula.

El presente texto rinde cuenta de una investigación sobre las articulaciones entre diversos elementos de inteligibilidad de las prácticas docentes, que emergen de un dispositivo de análisis y evaluación de lecciones, por una parte, y el hecho de que sean movilizados (o no) durante las lecciones, por otra parte. El estudio compara las prácticas de docentes generalistas (profesores de la educación básica primaria) y de docentes especialistas (licenciados en educación física o en artes) a quienes se invitó a manejar conjuntamente una unidad didáctica.

Los datos son el resultado de un análisis de este dispositivo en dos situaciones contrastadas:

- Dispositivo aplicado a la formación inicial de docentes, en el marco del manejo compartido de unidades didácticas en artes plásticas durante los períodos de pasantía del 2 do año de formación;

- Dispositivo aplicado a una investigación sobre el manejo en co-responsabilidad (generalista y especialista en educación física) de una unidad didáctica en educación física.

Metodológicamente, los datos provienen de la videoscopía y transcripción escrita de una lección de cada una de las dos secuencias. Nuestras categorías se basan en el modelo descriptivo de la acción docente propuesto por Mercier, Sensevy \& Schubauer-Leoni (2000), así como de los conceptos propuestos por Brousseau (1996) en el marco de su "Teoría de las situaciones didácticas". La participación de los docentes es fundamental en este dispositivo en la medida en que seguimos, igualmente, las propuestas sobre autoconfrontaciones cruzadas usuales en el campo de la psicología clínica del trabajo (por ejemplo, Clot \& Faïta, 2000), pero con las adaptaciones que hemos realizado desde hace algunos años en el campo de la formación inicial y continuada de los docentes (Goigoux, sous presse ; Lenoir \& ali, 2002).

Palabras clave. Autoconfrontación, gestos profesionales y evaluación formativa. 


\section{1.}

INTRODUCTION

Dans ce texte, nous souhaitons explorer les liens qui peuvent être établis entre l'évaluation formative en tant qu'outil institutionnel de régulation des processus d'apprentissage et des concepts didactiques (dévolution, régulation enseignante) tels abordés par la théorie des situations didactiques (Brousseau, 1996) ou la théorie anthropologique du didactique (Chevallard, 1999, 1995). L'objectif principal est celui d'interroger les rapports qui peuvent être établis entre les modèles, les concepts et les méthodologies de la recherche en didactiques, d'une part, et les démarches et dispositifs de formation des enseignants, d'autre part.

La question centrale est celle de savoir à quelles conditions et avec quels résultats les concepts et modèles construits dans un but de recherche descriptive du fonctionnement didactique peuventils être mobilisés dans les dispositifs de formation d'enseignants, tout en évitant le piège de l' "applicationnisme " pédagogique et didactique.

Cette étude se construit dans la conjonction des situations didactiques en tant que systèmes articulés d'enseignement/ apprentissages -entrée par l'analyse des pratiques et les théories de l'activité humaine- et, parallèlement, elle s'élabore sur la base des gestes professionnels de programmation, de gestion et d'évaluation de l'enseignant -l'activité enseignante comme analyseur des situations didactiques-.

De fait, de nombreux développements sont issus des didactiques disciplinaires telles que celle des mathématiques ou celle des langues, qui de par leur reconnaissance institutionnelle, ont connu une forte croissance depuis le milieu des années 1980. Ces travaux envisagent l'analyse des pratiques enseignantes dans une perspective de didactiques comparées (cf. Revue Française de pédagogie, 2002), dans le but de cerner la généricité de certains aspects liés à l'enseignement dans une discipline. Moins nombreux sont les travaux issus de didactiques disciplinaires dont la participation aux démarches de l'analyse descriptive du didactique ordinaire est plus récente, telles que la didactique des arts plastiques et/ou celle de l'éducation physique. Ces dernières développent actuellement des démarches d'ingénierie intégrées aux démarches de recherche (Brousseau, 1996) et font de l'activité enseignante un analyseur des systèmes didactiques ordinaires.

\section{PROBLÉMATIQUE}

Diverses modélisations du fonctionnement des systèmes didactiques ont émergé avec le passage, opéré durant les années 1980, d'une didactique prescriptive vers une didactique descriptive des activités d'enseignement (Schubauer-Leoni, 1998), notamment celle de la théorie des situations didactiques (Brousseau, 1986) basée sur la théorie des jeux, celle de l'approche anthropologique du didactique (Chevallard, 1991) et celle plus récente, de la théorie de l'action enseignante (Mercier, Sensevy \& SchubauerLeoni, 2000). Ces approches ont en commun une conception systémique et dynamique du fonctionnement des systèmes didactiques et tentent de produire des modèles capables de décrire et d'articuler les dimensions structurelle et fonctionnelle de l'activité didactique.

Dans le cadre d'une analyse des situations éducatives, la démarche descriptive en didactiques cherche à analyser les systèmes formels d'enseignement/apprentissage en lien avec les pratiques effectives qui se déroulent dans les institutions.

Cette approche met essentiellement en avant la nature dynamique des dispositifs d'enseignement/ apprentissage en ce qu'elle postule, dans le cadre de toute activité didactique, la mise en place et transformations d'un système de déterminations mutuelles:

enseignant 1 tâches d'enseignement/apprentissage 1 groupe-classe/élève.

Une des caractéristiques centrales des travaux récents issus de ces approches est sans doute le développement d'une conception résolument collective et dynamique de l'activité (didactique). Dans cette conception, les situations didactiques sont considérées comme résultantes de l'activité conjointe enseignant1 élève(s) (Leutenegger \& Ligozat, 2006; Mili \& Rickenmann, 2004 ; Rickenmann, 2006a). Cependant, il ne s'agit pas de considérer que ces agents partagent les mêmes buts, mais de se donner les moyens de penser l'interdépendance de leurs actions à l'intérieur d'un système dans lequel,

- l'action de l'élève est en partie contrainte par les choix de l'enseignant (choix des contenus d'enseignement, du type de tâche, du milieu didactique, de l'organisation sociale, etc...) ;

- l'action de l'enseignant est orientée en fonction des conduites potentielles et effectives de ses élèves.

Cette conception systémique et dynamique de l'activité conjointe, peut être décrite à partir de trois grandes familles de processus mis en évidence par Mercier, Sensevy \& Schubauer-Leoni (2000), qui sont à l'œuvre dans les systèmes didactiques ordinaires:

- Processus mésogénétiques : relatifs à la manière dont l'enseignant aménage un milieu d'apprentissages avec lequel les élèves interagissent et qu'ils font évoluer ;

- Processus topogénétiques : relatifs aux postures et rapports aux savoirs de l'enseignant et des élèves et leur évolution durant l'activité ;

- Processus chronogénétiques : relatifs à l'avancement du temps didactique et aux rapports entre les temps d'enseignement et ceux de l'apprentissage. 
Du point de vue de la recherche, les concepts de meso, topo et chrono-genèse présentent l'avantage de produire des descriptions dynamiques des phénomènes didactiques observables, c'est-à-dire, qui articulent l'« état " d'un élément du système didactique (enseignant, élèves, objets de savoir) par rapport aux autres, à un certain moment du déroulement de la tâche d'enseignement/apprentissage.

Comme nous tenterons de le montrer dans ce texte, sous couvert d'un dispositif d'évaluation formative, ces concepts pourraient également constituer un apport déterminant du point de vue de la formation aux gestes professionnels d'enseignement.

\section{L'ÉTUDE DESCRIPTIVE DE}

\section{L'ACTIVITÉ D'ENSEIGNEMENT}

La recherche sur les gestes professionnels de l'enseignant dans la perspective d'une didactique descriptive plutôt que prescriptive est relativement récente (Calderhead, 1995 ; Chevallard, 1995 ; Goigoux, 2000 ; Mercier, Sensevy \& Schubauer-Leoni, 2001 ; Portugais, 1992). Elle constitue le prolongement d'une longue tradition de recherches en pédagogie expérimentale, aussi bien aux EEUU (Biddle, Good \& Goodson, 1997) qu'en Europe (Crahay \& Lafontaine, 1989).

En sciences de l'éducation, ce sont essentiellement trois entrées qui abordent actuellement l'articulation des déterminations mutuelles des composantes des systèmes didactiques à partir de la notion d'activité -didactique-.

Une première entrée, praxéologique, propose un modèle théorique qui articule la tâche comme activité aux gestes afférents que les agents effectuent pour l'accomplir (Chevallard, 1995). Cette entrée constitue le développement du projet scientifique de fonder les didactiques disciplinaires, principalement la didactique des mathématiques, autour d'une réflexion sur les conditions de possibilité de la transmission des savoirs, en ce que ces derniers constituent des "objets vivants " évoluant à la fois à l'" extérieur ", dans la culture et à l' " intérieur » de l'institution scolaire (Brousseau, 1986).

Une deuxième entrée, celle des communautés de pratiques (Lave \& Wenger, 1991), propose une approche environnementaliste de la production collective et distribuée des savoirs. Moins sensible à la spécificité des objets de savoirs que la première, cette entrée fournit cependant un modèle descriptif des processus sociocognitifs centré sur les interactions, négociations et régulations de l'action entre agents au sein des situations d'enseignement/apprentissage. Dans cette entrée, ces dernières ont tendance à être considérées comme une version " micro " des situations socioculturelles -adidactiques- de production et de circulation des savoirs.
Empruntant la perspective historico-culturelle de Vygotski, les travaux d'une troisième entrée, sémiopragmatique (Sensevy, 2001; Mili \& Rickenmann, 2004; Rickenmann, 2001 et 2005), tentent d'articuler les apports des deux premières à partir de l'étude de l'objet enseigné d'une part et, d'autre part des gestes d'enseignement, en suivant l'évolution systémique et dynamique des dispositifs didactiques. Sensible au caractère propre de chaque discipline d'enseignement ${ }^{1}$, cette entrée prend en compte les phénomènes liés à la transposition interne des savoirs dans les situations didactiques. Sensible, aussi, à la nature co-construite, distribuée et conjointe des processus cognitifs, cette entrée considère également les formats didactiques, c'est-à-dire les types et modalités d'organisation des tâches d'apprentissage propres à l'institution scolaire et les effets qu'ils ont sur l'activité des agents -enseignant/élève-s-, sur la construction des objets effectivement enseignés (Wirthner \& Schneuwly, 2004).

Les travaux centrés sur les deux premières entrées ont permis de développer, particulièrement, les connaissances relatives aux composantes savoir(s)/tâches d'enseignement et sur les processus d'apprentissage. En ce qui concerne l'action enseignante, ce sont les travaux de la troisième entrée qui abordent la description des systèmes didactiques " ordinaires » à partir d'une étude des gestes de l'enseignant en situation d'enseignement effectif (Dévolvé, 2000; Mercier, Sensevy \& Schubauer-Leoni, 2000; Salin, 1997).

\subsection{L'ACTION ENSEIGNANTE COMME ANALYSEUR DES SITUATIONS \\ DIDACTIQUES}

Dans le cadre de cette démarche, les didactiques AP et EP ont recours aux conceptualisations et démarches méthodologiques sur l'action enseignante produites, notamment, par les travaux en didactiques des mathématiques et des langues.

Ces travaux proposent, d'une part, des catégories fonctionnelles de l'action enseignante, relatives à son rôle fondamental de médiateur des processus d'enseignement/apprentissage: définir, dévoluer, réguler et instituer se présentent ainsi en tant que catégories favorables à l'identification des différentes actions de l'enseignant et des fonctions qui s'y rapportent. Ces catégories facilitent ainsi le découpage séquentiel du continuum de l'activité enseignante, ainsi que son analyse en fonction du caractère finalisé -transmettre des savoirs-, de la situation didactique. Articulées aux concepts de mésogenèse, de topogenèse et de chronogenèse, ces catégories permettent également d'analyser le rôle, les fonctions et effets de l'action enseignante aux prises avec le système didactique effectivement à l'œuvre et puis, son évolution.

La focalisation sur l'enseignant pour l'étude des systèmes didactiques, en particulier en ce qui concerne la gestion de l'activité, les décisions qu'il prend tout au long de l'évolution de la tâche et les différents topos qu'il assume, rend compte de l'influence mutuelle entre les types de savoirs, les formats d'activité didactique et l'agir interdépendant de l'enseignant et des élèves.

\footnotetext{
1 Pour une revue analytique sur l'historique et constitution des différentes démarches adoptées par les didactiques disciplinaires cf. Bronckart, 2005; Sieber-Thévenaz, 2005.
} 


$$
\text { (2) }
$$




\subsection{MÉTHODES CLINIQUES D'ÉTUDE DE} L'ACTION ENSEIGNANTE

Parmi les méthodes auxquelles ont recours les chercheurs pour l'analyse de l'action enseignante, le recours aux techniques d'autoscopie ont souvent permis de mettre en évidence les dimensions de l'activité didactique liées à sa mise en œuvre effective. Issues, notamment, des techniques d'autoconfrontation croisée de l'analyse du travail (Clot \& Faïta, 2000), différents dispositifs d'autoscopie ont actuellement cours dans les travaux de recherche, le plus souvent complémentaires des dispositifs plus classiques de l'analyse didactique tels que la vidéoscopie des séquences d'enseignement et leur mise en protocole écrit.

\section{PREMIER DISPOSITIF (EP) : AUTOCONFRONTATIONS CROISÉES, RECHERCHE ET RÉGULATION}

À travers le premier dispositif que nous allons vous présenter, nous souhaitons montrer quelques moments d'un processus conjoint d'auto-évaluation des enseignants. Pour une question de place, nous nous centrerons ici sur l'évaluation globale qu'ils effectuent de l'ensemble de l'unité d'enseignement-apprentissage sur le basket-ball $(5 \mathrm{P})$, démarche qui les amène à évoquer les apprentissages des élèves et à questionner la pertinence de leur programmation en analysant le travail de construction et de mobilisation des savoirs effectué par les élèves (des petits jeux à la situation de match) et les moments de verbalisation sur lesquels repose une partie de la programmation.

Cette étude fait partie d'une recherche en cours qui a comme but la description et la compréhension de l'action didactique des maîtres dans l'enseignement de l'éducation physique à Genève. Les caractéristiques particulières du fonctionnement de cette discipline dans ce canton nous ont amenés à nous intéresser à une double perspective d'investigation. D'une part, la relation didactique qu'entretiennent le maître et le(s) élève(s) au cours des séances ordinaires (Leutenegger 1999), ce qui nous a conduits à observer et à filmer plusieurs leçons en salle. Nos préoccupations rejoignent ici le questionnement développé en France depuis une vingtaine d'années à propos de l'action de l'enseignant (Amade-Escot et al., 1998) et tout particulièrement des travaux récents en didactique de l'éducation physique (Amade-Escot, 2002, 2003, Loquet et al., 2002, Thepaut, 2003).

D'autre part, nous nous intéressons à la relation didactique que construisent le maître spécialiste (MS) et le maître généraliste (MG) dans l'enseignement conjoint et en alternance ${ }^{2}$ (co-responsabilité) de l'EP et à la progression du savoir au sein de l'unité d'enseignement/apprentissage.

Pour tenter de mieux comprendre l'action de ces enseig-

\footnotetext{
2 A l'école primaire Genevoise, le maître spécialiste a la fonction de « méthodologue ». En tant que tel, il a la responsabilité de la programmation annuelle et une place centrale dans le choix des contenus et des tâches à aborder avec les élèves. C'est lui le responsable de la 1ère séance de l'unité d'enseignement/apprentissage. Ensuite, le MG est sensé poursuivre le travail du spécialiste durant deux séances, avant que ce dernier revienne dans la classe pour donner une autre leçon. En moyenne, les unités sont composées de 6-7 séances (2 pour le MS, 4-5 pour le MG).
}

nants, nous avons adapté un outillage méthodologique utilisé avec d'autres catégories professionnelles et sensé " créer les conditions d'un rapport dialogique " (Faita et Viera, 2003) entre les deux acteurs. En effet, à l'issue des séances filmées, les deux enseignants concernés ont participé à un entretien d'autoconfrontation croisée. C'est à partir de cet entretien, et par le regard posé par chaque maître sur sa propre pratique et sur celle de son collègue et, concurremment, sur leur participation/articulation aux apprentissages des élèves, que nous tentons de comprendre la dynamique des acteurs et leur action dans le système didactique.

$\mathrm{Au}$ niveau méthodologique, soulignons simplement que les séquences visionnées lors de l'entretien ont été choisies par le chercheur, en assumant ainsi la responsabilité d'extraire des séances les dimensions pertinentes de l'action des enseignants ${ }^{3}$, et préalablement visionnées par les maîtres chez eux $x^{4}$.

De même, et cela constitue une des particularités méthodologiques de la recherche, la structuration temporelle des leçons a été modifiée en plaçant au début du DVD la situation de jeu réel (basket dans tout le terrain), situation qui clôturait la dernière séance de chaque maître. Notre choix de confronter en premier lieu les enseignants au visionnement de la tâche finale, qu'ils considèrent comme étant la situation de jeu la plus complexe et potentiellement mobilisatrice des savoirs abordés précédemment, a produit des effets, comme nous allons le voir plus bas, dans la réflexion et le discours des maîtres.

Les autres tâches du support respectent la chronologie des séances et ont été retenues au regard de trois critères : a- un lien évident avec les contenus propres au basket (nous n'avons pas retenu, par exemple, les moments d'échauffement sans ballon ou le travail d'adresse individuelle), b- des moments pouvant être considérés comme centraux dans la démarche d'enseignementapprentissage des maîtres (ex. : les verbalisations), c- la présence de la même tâche chez les deux maîtres.

\footnotetext{
3 C'est au « chercheur, partiellement, parfois totalement, d'envisager le choix de moments et séquences de l'activité en fonction de cette activité - plus exactement de la perception qu'il en a - plus que de la représentation discursive que le collectif est disposé à en fournir. » (Faita \& Viera, op. cit.; Rickenmann, 2006b)

4 Quinze jours avant l'entretien, nous avons donné aux maîtres un DVD avec les différentes séquences. Sur papier, nous leur avons fourni une explication du choix effectué ainsi que 4 points pouvant être pris en considération lors du visionnement (1. Selon moi, de quelle manière sont organisées les différentes tâches et leçons de l'unité d'enseignement/apprentissage ?,2. Qu'est-ce que je peux dire par rapport à l'articulation de mon enseignement avec celui de ma collègue ?, 3. Quelles questions est-ce que j'aimerais poser à ma collègue pour mieux comprendre son enseignement ?, 4. Si je devais préparer un autre cycle de basket, comment m'y prendrais-je?
} 


\subsection{L'ÉVALUATION DES APPRENTISSAGES COMME DÉCLENCHEUR DE}

\section{L'ÉVALUATION DE L'ENSEIGNEMENT}

Le premier extrait que nous présentons provient du début de l'entretien. Le MS se positionne par rapport aux séquences choisies par le chercheur et formule une première évaluation de l'unité d'enseignement-apprentissage.

29- C : Est-ce que vous souhaitez dire quelque chose avant de passer au visionnement ? Toi, tu avais déjà réagi par rapport au choix...

30- MS : Comme tu avais mis le match d'abord

\section{1- C : oui (en accompagnement du discours)}

32- MS : et après on voit les séquences, je me disais, du coup euh, en fait, j'ai fait une mise en place, donc quand j'ai construit mon truc,

\section{3- $\mathrm{C}$ : tout à fait (en accompagnement du discours)}

34- MS : j'ai commencé par les jeux, il faut faire ceci et après cela, pour arriver au match. Donc, du coup, c'est vrai qu'on voit la situation finale, mais du coup on sait pas, ben, moi, ce qui m'a gêné c'est que...on ne savait pas tout ce que j'avais mis en place pour arriver au match, donc c'était juste comme ça.

35- $\mathrm{C}$ : Et donc, si tu devais dire la progression que tu as mise en place, comment elle est faite cette suite

36- MS : Oui, alors, alors moi, ce qui m’a frappé, je le disais à Nadia, c'est qu'on a mis des choses en place, mais en fait, finalement dans le match, les enfants ils sont pas fait le lien du tout avec tout ce qu'on avait fait. Ils ont joué, en fait, on aurait pu faire le match sans faire tout ce qu'on a fait avant (sourire) et moi j'ai l'impression qu'ils n'auraient pas plus mal joué, quoi, ou mieux joué.

Le MS ne s'y retrouve pas dans les choix effectués par le chercheur et évoque la logique de progression de leur enseignement, celle qui a animé les 6 séances. Il constate que, par l'absence des tâches initiales qu'il considère comme essentielles, cette logique n'est pas prise en considération ou reconnue en tant que savoir professionnel ("...ce qui m’a gêné c'est que... on ne savait pas tout ce que j'avais mis en place pour arriver au match..."). Ce visionnement à l'envers ("on voit la situation finale mais du coup on ne sait pas ") produit, en même temps, une prise de distance chez l'enseignant qui l'amène à porter un regard global sur l'ensemble des séances et à évaluer le travail réalisé. La situation de match devient ainsi révélatrice des acquisitions des élèves et, du même coup, de leur propre enseignement. 
37- C : Mais, qu'est-ce qu'il leur manque dans la situation de match ?

38- MS : Ils arrivent pas à, à

39- MG : (simultanément) Mettre en (XXXX)

40- MS : à mettre en pratique ce qu'on a fait avant, pour eux c'était autre chose. Je remarque ça dans la natation, quand on leur dit de nager librement, ils, ils nagent n'importe comment

41- MG : oui, oui (simultanément)

42- MS : tu leur fais faire des exercices pour corriger la respiration, ils font très bien et après, tout à coup, tu leur dis bon, nagez...ils nagent comme des petit chiens

43- MG : On a le même problème en en, par exemple en français, euh, dans les didactiques. Des exercices séparés, ils les font justes, mais après en pratique lorsqu'ils doivent écrire un texte, ça, ils ne font pas

44- MS : Je ne sais pas si c'est l'âge, si c'est...je ne sais pas

45- MG : Moi je pense que ces exercices, quand même de, de technique qui sont importants, que ça soit pour (phrase interrompue)

46- MS : On a fait peu de technique, on a justement fait des, des, des, des mises en situation, j'ai l'impression après qu'ils transposeraient plus facilement, mais ce que je remarque c'est qu'ils ne transposent rien du tout

(...)

81- MS : Cath : Mais, bon ça, je crois que c'est euh comme, si tu dis qu'en français c'est la même chose, je le remarque en natation, c'est vraiment flagrant

82- MG : Mais je crois qu'au bout d'un moment ça fait le déclic, mais peut- être pas au premier match. Mais je crois que euh, au bout d'un moment, à force de jouer, ils se souviennent des exercices, des petites choses qu'ils ont faites et puis euh...ça leur permet de progresser.

Le MS attribue l'absence de transposition à l'incapacité des élèves à (ré)utiliser les savoirs abordés dans les différents « exercices " préparatoires. En même temps, il voit qu'une action de sa part pour maintenir la mémoire didactique pourrait rendre plus efficace les moments de verbalisation (TdP 51 : "Alors, moi ce que je ferais si je refais l'année prochaine, enfin, la prochaine fois que je refais du basket, je pense que je leur dirai...vous vous rappelez de ce jeu, qu'est-ce qu'on cherchait dans ce jeu?"). Pour le MG, qui considère l'assemblage des parties ("des exercices séparés") dans un tout comme relevant d'une problématique plus générale et semblable aux autres didactiques , ("On a le même problème en en, par exemple en français... "), le temps didactique expliquerait le niveau atteint par les élèves (TdP 82).

Cette interprétation, bien qu'intéressante par la prise en considération du temps qui serait nécessaire aux transformations des conduites motrices, va clore le débat sur ce sujet et nous 
paraît, par son caractère restrictif, empêcher les maîtres de questionner les objets effectivement traités, le type de tâches agencées et leur rapport avec les compétences nécessaires pour faire du basket.

L'analyse a priori des tâches que nous avons réalisée montre que la plupart d'entre elles ont un rapport de contextualisation très faible ${ }^{5}$ (ex. : absence d'adversaire ou de terrain orienté) et que la transposition attendue repose, en grande partie, sur un savoir procédural construit lors des moments de verbalisation, moments qui structurent et jalonnent l'ensemble des séances.

Pour montrer cette démarche de prise de conscience et de verbalisation instaurée par les enseignants, nous allons présenter des extraits tirés de la lère leçon du MS et de l'entretien cité plus haut. Ils nous paraissent représentatifs des techniques topogénétiques et mésogenétiques qu'ils ont utilisées pour solliciter, valider et instituer des règles d'action (savoirs à mobiliser dans le jeu réel) et permettent, là aussi, d'appréhender la particularité de leurs rapports au savoir.

\subsection{LES DISPOSITIFS D'ENSEIGNEMENT DE SO- LLICITATION \\ RÉFLEXIVE}

Lors de la première leçon et après l'échauffement, le MS définit les règles des trois jeux auxquels les élèves vont jouer ( $T d P$ 41: "Sur ce terrain on va dire que ce sont les rouges en premier qui sont les attaquants, donc ils auront le ballon et ils vont essayer de se faire des passes ") et dévolue la tâche tout en leur proposant des pistes pour réussir.

Ce " vous allez voir " peut être considéré comme une $c l \hat{o}-$ ture topogénétique (Sensevy et al. p. 290). L'enseignant dit aux élèves, sans le développer explicitement, qu'il existe une stratégie optimale et qu'à partir de ce moment, c'est à eux de trouver la solution. En même temps, sa formulation nous semble renforcer la procédure transmise aux élèves par questionnement (pour défendre, on ne reste pas tout le temps sur le tapis) et évoquer implicitement les contraintes de la tâche auxquelles ils seront confrontés (le nombre de tapis est plus grand que le nombre de joueurs). Pour le MS, les contraintes revêtent la fonction de déclencher le processus adaptatif et créatif des élèves. En disant " vous allez voir ", on prévoit également une future validation de la part de l'enseignant par de traits pertinents (TdP 53) et une action d'instituer une règle d'action que nous considérons, en regard des matériaux récoltés (transcriptions des entretiens et séances vidéoscopées) comme un des contenus principaux visés.

5 Lors d'une autre réunion, nous avons présenté au MS le contenu des interactions didactiques maître-maître C'est à ce moment qu'il a pu porter un regard différent sur la nature des tâches de la programmation et comprendre autrement les résultats de leur action. Manifestement, il éprouvait des difficultés à s'éloigner de son rapport habituel au savoir et aux déterminations institutionnelles, mouvement facilité par l'apport de savoirs didactiques qu'il n'avait pas à disposition.

53- MS : Pas le droit de marcher, avec le ballon mais sans le ballon, évidemment, tu vas devoir tout le temps, tout le temps bouger, parce que vous n'allez pas pouvoir rester comme ça (il se place immobile, pieds écartés), la même chose est-ce que ça vaut la peine (il se dirige vers le tapis) pour un défenseur (il est sur le tapis) de rester tout le temps sur ce tapis?

54- Abdel: Non

55- MS: Vous allez voir. 


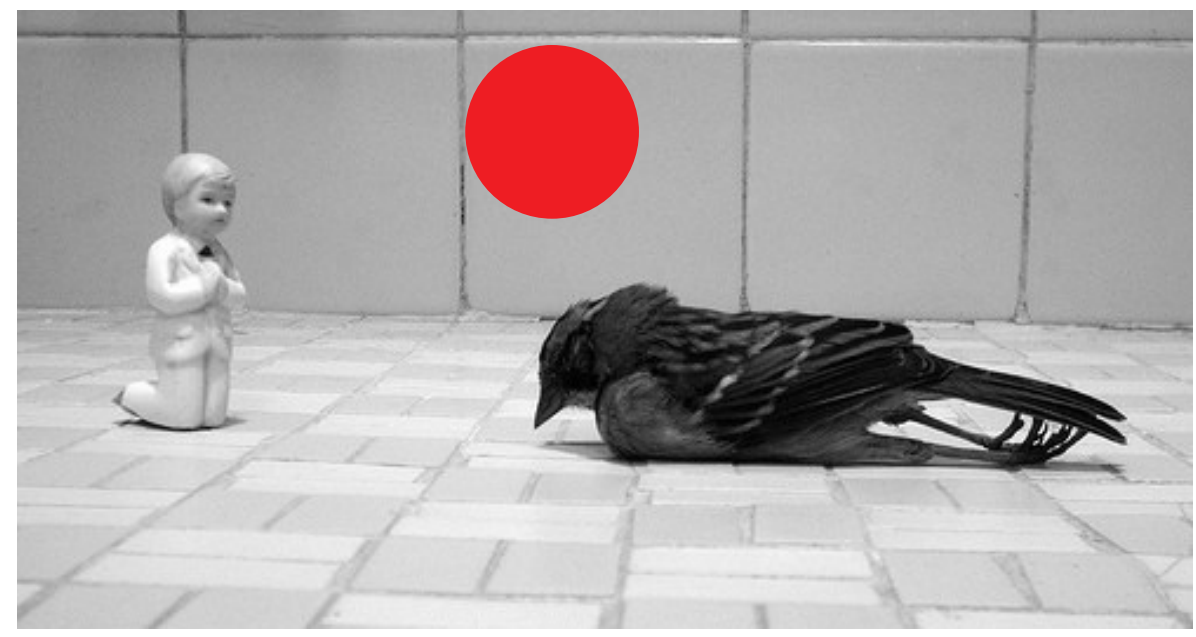

\begin{tabular}{|l|c|}
\hline Obra/Titulo: & xxxxx Xxxxxxxx \\
\cline { 1 - 2 } & Laura Duque Quinones \\
\hline
\end{tabular}

La situation de jeu est suivie d'un moment de verbalisation ${ }^{6}$ qui permet au maître de savoir si les élèves ont bien vu. Ce moment débute par la sollicitation de l'enseignant d'une position réflexive des élèves et cela par un questionnement implicite (MS : "Si vous deviez donner un conseil (...) pour aider vos camarades à bien jouer tout de suite »). Durant tout l'échange, il adopte une posture de surplomb topogénétique en évaluant et statuant la pertinence de ce qui est dit, ceci lui permet de faire désigner aux élèves ( $\mathrm{El}$ : " il faut marquer son joueur ») ou de désigner lui-même ce qui est vrai et d'instituer, en les rendant publiques, les repères (règles d'action) que les élèves doivent prendre en considération lors des situations de jeu qui vont suivre (MS : "...ça paraît plus difficile de surveiller les tapis que surveiller chacun son joueur »). Ce processus, qui s'apparente à la structure générale dialogique et tripolaire évoquée par Sensevy et al. (op. cit., p. 282) est emblématique du fonctionnement des deux enseignants.

Lors de l'entretien, ils y reviennent en faisant référence aux situations de jeu réel (match de basket en grand terrain) durant lesquelles les maîtres ont demandé aux élèves d'adopter, quand ils sont sur les bancs, un topos différent : l'élève n'observe/parle plus de sa pratique mais est amené à analyser, en adoptant la posture d'observateur (journaliste), la façon de jouer des équipes sur le terrain ("Donc vous vous allez regarder que ça dans le jeu, les verts comment ils défendent, vous les rouges, comment ils se font des passes, comment ils s'organisent pour aller à l'attaque, ça marche?).

Les moments de verbalisation qui suivent les matchs commencent aussi par une sollicitation topogénétique (ex.: "Messieurs, mesdames les journalistes qu’avez-vous observé ?") qui constitue le début du processus dialogique évoqué plus haut. Les enseignants vont, en validant la pertinence des avis émis, ou en reformulant l'énoncé des élèves, définir le projet d'action pour les parties suivantes (ex.: "vous choisissez dès le début de la partie, le joueur que vous allez marquer ").

Durant l'entretien, les enseignants analysent cette partie du dispositif. Leurs avis se rejoignent dans l'évaluation de la qualité des observations/interventions des élèves ("leurs remarques, elles étaient, je les ai trouvées bonnes"), mais divergent au moment de comprendre l'impact au niveau des apprentissages effectifs.

6 L'organisation des séances a induit chez les élèves une sorte « d'attente » de moments de verbalisation. Il suffisait à l'enseignant de siffler la fin d'un jeu pour que les élèves lèvent automatiquement la main, sachant à l'avance qu'ils seraient questionnés. 
469- MS : Je trouve qu'ils avaient bien vu le jeu. Mais où j'étais déçu c'est qu'ils...ils voyaient bien, mais après, quand ils jouaient, ils arrivaient pas à, à tenir compte de ce qu'ils avaient pourtant vu, quoi. Moi je trouve que ça serait plus utile ces moments euh pour jouer.

470- C : Donc, verbaliser, dire, ça serait utile pour jouer.

471- MS : Oui, moi je pensais que ça serait, le fait de verbaliser, d'avoir observé, d'avoir vu les fautes des autres, je pensais que ça leur serait utile à eux pour le jeu.

472- MG : En même temps, c'est super difficile, parce qu'une fois que toi tu es dans le jeu, je veux dire

473- MS : (simultané) Oui

474- MG : tu joues donc le, le fait de réfléchir aux erreurs des autres et de tenir compte

475- MS : (simultané) Oui, oui

476- MG : de ces erreurs pour les mettre en pratique...même nous, en tant qu'adultes je, je ne suis pas sûr que ça marche vraiment

477- MS : Donc, justement, est-ce que c'est finalement euh

478- MG : (simultané) Je crois qu'il faut le répéter, le répéter, le répéter.

479- MS : Parce qu'à ce moment-là c'est vraiment si utile que ça, mais moi c'est aussi parce que, comme les élèves sont un moment immobiles sur un banc, moi je n'ai pas envie qu'ils passent leur temps à rigoler entre eux et à pas s'occuper de ce qui se passe, puisque je pense qu'on apprend quand même en observant

480- MG : (simultané) Je crois que c'est utile quand même

481- MS : je crois que c'est aussi leur donner quelque

482- MG : Quelque chose à faire

483- MS : à faire et pas rester et pas rester

484- MG : Je crois que c'est vraiment utile mais sur euh, euh, sur le long terme 485- MS : Oui

486- MG : pas sûr euh, par sûr, d'un match à l'autre, en fait. Mais je pense qu'à force de répéter ces choses... 
Dans cet extrait, le MS revient aux situations de match et, en les considérant comme des moments d'actualisation des savoirs évoqués précédemment ("mais après, quand ils jouaient "), il questionne l'intérêt des verbalisations. Le MS constate que la capacité à bien voir le jeu (topos observateur) et à " verbaliser " (savoir procédural) n'est pas corrélative, pour autant, d'une transposition sous forme de savoir en action ("ils arrivaient pas à, à tenir compte de ce quils avaient pourtant vu »). Son analyse le conduit à envisager de transformer le temps de verbalisation en temps de jeu ("Moi je trouve que ça serait plus utile ces moments euh pour jouer") et à dévoiler d'autres raisons concernant la mise en place du dispositif comportant des observateurs, celle-ci plus générique et en lien avec la gestion du groupe-classe (TdP 479, 480-481).

La position du spécialiste contraste avec l'avis du MG qui considère que le niveau atteint par les élèves et, par conséquent, la transposition qu'ils ont réalisée, sont à comprendre au regard de la complexité du passage du savoir procédural au savoir en action ("ćest super difficile", " même nous, en tant qu'adultes ") et au besoin d'un temps pour apprendre plus important que celui de l'unité mise en place (TdP 478, 484, 486). Les références des enseignants se distinguent et montrent à nouveau des assujettissements différents en termes de rapport au savoir.

\subsection{POUR CONCLURE PROVISOIREMENT}

L'entretien d'autoconfrontation croisée que nous avons mis en place devait permettre, à travers le rapport dialogique des acteurs, incité par le visionnement de séquences choisies, de favoriser l'émergence des réflexions concernant l'enseignement conjoint et en alternance de l'éducation physique. Les extraits présentés nous paraissent montrer une certaine distanciation des enseignants envers leur pratique et le potentiel formateur du dispositif, sans que l'on puisse pour autant présager, à partir de ce retour réflexif, des changements dans leur manière de concevoir et de faire les leçons.

Nous constatons l'assujettissement des maîtres au fonctionnement habituel de la dyade qu'ils forment. La confrontation, dans ce sens, nous a paru être influencée par les repères partagés, limitant sûrement ainsi le questionnement de leurs dispositifs et modèles didactiques. Ceci interroge l'absence (voulue) dans le dispositif, de propositions ou de références extérieures au système étudié (introduites par le chercheur), repères par exemple didactiques concernant les contenus d'apprentissage qui seront (voir par exemple Sensevy \& Mercier, 2003) ou ont été abordés durant l'unité. Ces références nous semblent être porteuses potentielles d'un autre niveau de décentration et d'analyse et être plus enclines à répondre aux problématiques qu'émergent des démarches de formation des enseignants.

Avec le deuxième dispositif que nous vous présentons, nous poursuivons notre réflexion et nous tentons, dans un contexte bien différent (formation initiale) de comprendre les effets que produit dans la construction de gestes professionnels chez les étudiants, un dispositif d'autoconfrontation outillé par des connaissances didactiques sur l'action de l'enseignant et sur le contenu d'enseignement (Peindre à la manière de Pollock).

\section{DEUXIÈME DISPOSITIF (AP): VIDÉOS- COPIE ET RÉGULATIONS DE L'ACTIVITÉ D'ENSEIGNEMENT}

Comme nous venons de le montrer, les dispositifs basés sur l'autoconfrontation croisée recèlent potentiellement des processus d'explicitation et de distanciation analytique de la pratique enseignante, qui peuvent être mobilisés également dans le cadre de la formation professionnelle des enseignants.

Dans le cadre de la formation des enseignants du primaire à Genève, notre équipe de recherches mène depuis deux ans un travail d'analyse de ce type de dispositif, que nous utilisons, en tant que formateurs en didactiques des disciplines, pour instrumenter les démarches d'évaluation formative de nos dispositifs de pratiques sur le terrain scolaire. Dans le cadre de ce texte, nous souhaitons présenter quelques éléments résultant de ce travail en soulignant, toutefois, qu'il ne s'agit ici d'avancer que quelques premiers éléments d'une problématique émergente dans le champ de la recherche en didactiques (cf. Goigoux, 2000).

Comme nous l'avons annoncé au début, notre dispositif de formation se base sur l'étude de l'action enseignante comme analyseur des pratiques de formation. La vidéoscopie des leçons et l'autoconfrontation des stagiaires à leurs pratiques de terrain passe par un travail d'instrumentation didactique pour la formation qui nous semble répondre à quelques-unes des limites du dispositif tel qu'utilisé pour la recherche et soulignées précédemment.

La formation des enseignants à Genève est basée sur une structure d'alternance entre les semaines universitaires (cours, séminaires et ateliers) et des semaines de terrain durant lesquelles les étudiants sont amenés à prendre en charge, en dyades, l'enseignement des disciplines des modules didactiques. Résolument tourné vers une intégration des apports théoriques et de divers outillages de gestion et d'analyse des pratiques, notre dispositif cherche à travailler autant la dimension de planification (séquences didactiques de 2 à 4 séances de 50 min sur deux semaines), que celle de gestion en responsabilité des enseignements. Durant les semaines universitaires qui précèdent les terrains, demandons à nos étudiants de produire, en dyades, des séquences planifiées autour d'un sujet appartenant au programme de la classe qui les accueille, en accord avec l'enseignant titulaire qui joue le rôle central de formateur de terrain. Outre la planification des leçons, les dyades doivent filmer entièrement leurs interventions, avec répartition de rôles : un étudiant mène une ou deux leçons alors que l'autre se limite à le filmer et à observer la pratique du premier. La semaine de terrain qui suit, les rôles sont inter-changés à l'intérieur de la dyade de manière à ce que chaque étudiant ait l'opportunité de travailler les deux postures. 
Obra/Titulo: $\quad$ xxxxx Xxxxxxxx

Autor/registro: Paola Camargo

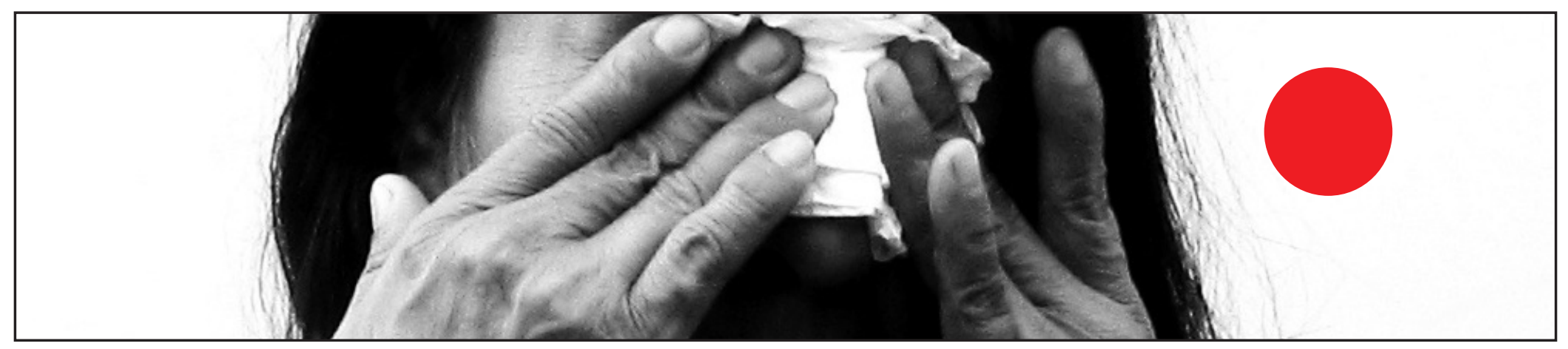

FALTA TEXTO DE OBRA DE LA ARTISTA 
Obra/Titulo: $\quad$ xxxxx xxxxxxxx

Autor/registro: Paola Camargo

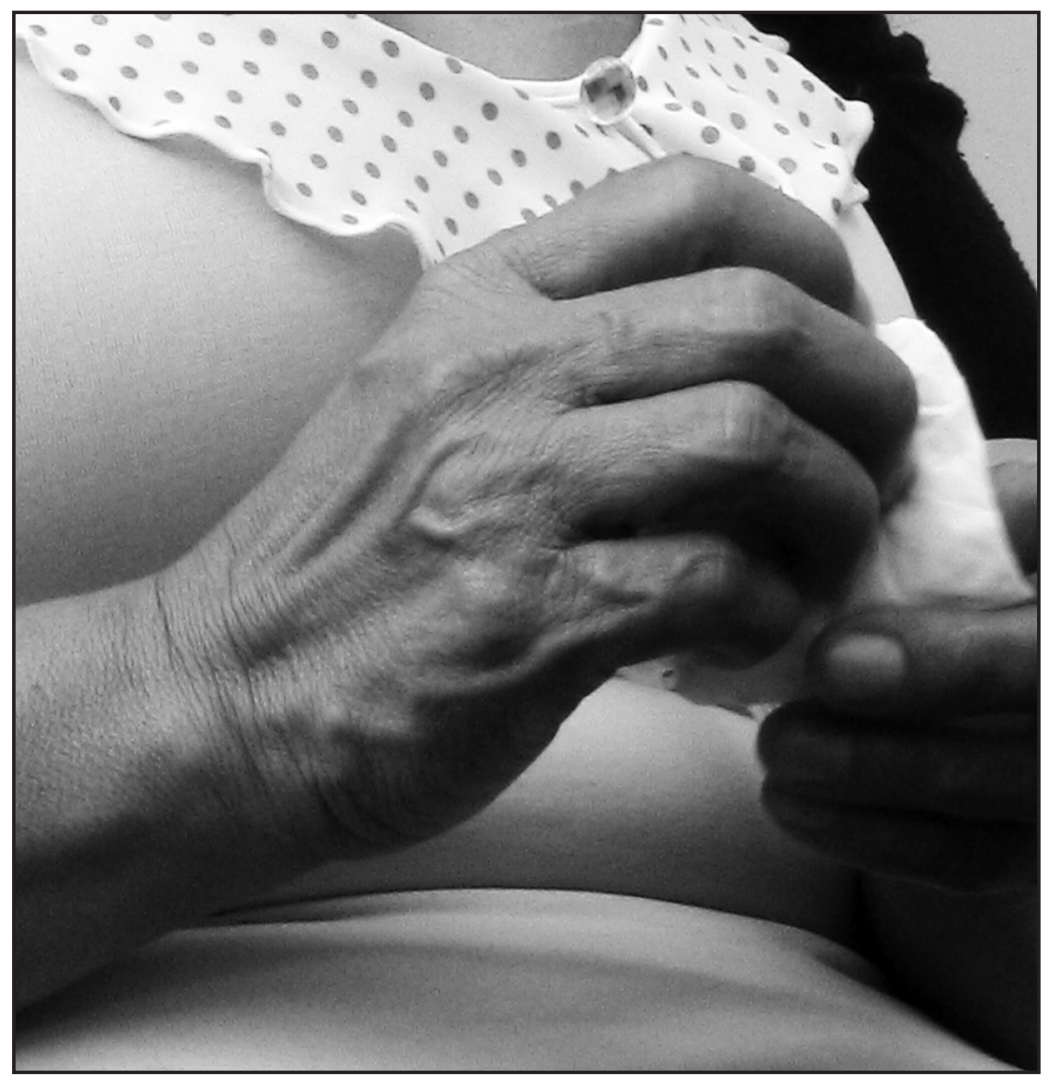

FALTA TEXTO DE OBRA DE LA ARTISTA 
Le dispositif d'autoconfrontation croisée intervient entre la deuxième et la troisième semaine de terrain. Les étudiants sont invités à analyser leur pratique en cherchant à identifier des phénomènes liés à l'un des trois processus méso-topo ou chrono/génétiques; puis, ils sont incités à investir les résultats de cette analyse dans la suite des pratiques de la troisième semaine. L'objectif du dispositif est que les étudiants arrivent à développer ou, le cas échéant, à remédier, certains de leurs gestes d'enseignement.

\subsection{UN EXEMPLE : LA MODIFICATION DE LA GESTION TOPOGÉNÉTIQUE DANS UNE LEÇON * FAIRE À LA MANIÈRE DE POLLOCK » (3P ET 5P)}

Les étudiants ayant choisi un sujet assez fréquent dans l'enseignement des arts plastiques (Peindre à la manière de J. Pollock et/ou peindre avec la technique du dripping), nous avons pu constituer un petit dispositif de recherche, dont voici les principales caractéristiques:

- Constitution d'un "groupe témoin " (GT) à partir de la vidéoscopie des pratiques d'un enseignant spécialiste ayant mené une séquence de trois séances auprès d'un groupe d'élèves de double degré (4ème et 5ème primaire). Les trois séances ont été planifiées et gérées sans intervention des chercheurs et constituent, dans le cadre de notre recherche, un groupe témoin représentatif des pratiques de l'enseignement ordinaire.

- Quatre groupes expérimentaux avec des élèves de 1ère (1 gr.), troisième (1gr.) et quatrième (2 grs.) primaire, auprès desquels quatre dyades ont réalisé leurs pratiques de terrain (ci-après, groupes de stage ou GSta).

- Un travail de planification des séquences autour du thème " peindre à la manière de... ", en dyades selon la démarche explicitée plus haut ;

- Une première phase d'autoconfrontations entre la deuxième et troisième semaines de terrain, avec une durée minimale d'une heure. Cette séance a été instrumentée à l'aide des trois catégories de phénomènes didactiques (méso, topo ou chrono/génétiques), que la consigne du formateur universitaire les a invité à mobiliser pour l'analyse (analyse de leur pratique à partir de l'un des trois types de processus);

- Un retour sur le terrain, avec des régulations concernant la planification et/ou la gestion de l'enseignement, basées sur la séance d'autoconfrontation. Ces pratiques ont été également vidéoscopées ;

- Une deuxième séance d'autoconfontations, cette fois en présence du chercheur, à partir d'extraits, parmi l'ensemble des leçons données, considérés comme significatifs par les étudiants.

- Dans le cadre de la recherche, les étudiants ont été invités à regarder également la séquence du $\mathrm{GT}^{7}$ entre la deuxième et troisième semaines de terrain.

Du point de vue de la structure des leçons, les étudiantes qui ont comparé, lors de la deuxième séance d'autoconfrontations,

7 Cette dernière étape ne fait pas partie du dispositif de formation habituel les leçons Gsta avec la leçon GT, ont constaté quelques différences significatives du point de vue des gestes professionnels d'enseignement.

Une différence, identifiée par les étudiantes comme plutôt négative du point de vue de leur propre travail, consiste en une certaine « rigidité » dans la structure de la séquence GSta par rapport à celle du GT (voir tableau en fin de texte). Cette rigidité a été constatée, notamment, par rapport à une division beaucoup plus évidente dans la leçon Gsta entre les phases appartenant au travail de l'enseignant et celles considérées comme des phases dévolues au travail de l'élève. Un des observables utilisés par les étudiantes a été leur analyse de la première tâche de la leçon 1, dont la fonction était celle de faire " un petit apport théorique sur J. Pollock et sa peinture ». Cette première tâche était conçue par les étudiantes comme relevant essentiellement de l'enseignement, avec une activité des élèves réduite à une écoute attentive. En ce sens, un des effets positifs de l'autoconfrontation a été la proposition de rémédiation réalisée durant la troisième semaine de terrain, avec l a production d'une " première tâche ", ayant gardé la fonction d'introduction à la peinture de Pollock, mais travaillant davantage sur les topos des élèves. C'est ainsi que la deuxième étudiante a posé davantage de questions, a invité ses élèves à observer et à comparer des reproductions de Pollock ; durant cette première tâche, les informations sur Pollock ont davantage été produites à partir d'informations proposées par les observations des élèves.

Une autre différence soulignée par les étudiantes, cette fois-ci comme positive, a été le constat d'une richesse topogénétique beaucoup plus grande dans leur propre séquence que dans celle du GT. En effet, dans le séquence du GT, la posture d'apprentissage privilégiée a été celle de "l'artiste producteur ", accompagnée par une deuxième posture de verbalisation (troisième leçon) ; par contraste, la leçon GSta développait une variété de postures plus importante, basée sur une vision plus large des pratiques sociales de référence (voir tableau en fin de texte): posture d'observateur (tâche 1, leçon 1), posture de production mais articulée à une modalité de travail collaboratif (tâche 2, leçon 1), posture critique (activité 1, leçon 2), posture de verbalisation (tâches 1 et 3 , leçon 3),...

Du point de vue de la gestion des leçons effectives, les étudiantes ont choisi de mener une analyse de quelques phénomènes topogénétiques relatifs aux tâches de la deuxième semaine de terrain.

Les résultats de cette analyse ont été centrés sur les gestes et/ou actions de "dévolution et régulation » de l'activité des élèves. Par rapport aux leçons GT, les étudiantes ont constaté leur difficulté à produire des gestes de dévolution effective. L'autoconfrontation a permis de mettre en évidence le fait qu'elles n'avaient pas "vraiment écouté» les réponses d'élèves aux questions émergeant de l'interaction enseignant-élève ; ainsi que le fait qu'elles ont eu tendance à fournir souvent les 
réponses à la place des élèves, dans un souci de faire avancer la leçon. Par exemple, durant la deuxième tâche (leçon 1), les étudiantes ont constaté qu'au bout de cinq minutes seulement, l'une d'entre elles avait déjà " poussé " ses élèves à produire l'ensemble des gestes picturaux attendus (se déplacer autour de la feuille, changer les positions du corps, contrôler l'amplitude du geste, etc...). Autrement dit, elles ont constaté que, sous la pression de leur propre contrat de formation ${ }^{8}$, la stagiaire n'avait pas laissé le temps aux élèves pour interagir avec le milieu didactique proposé.

Fruit de ces analyses, nous avons pu observer des effets positifs lors de la reprise de la séquence en troisième semaine de terrain. Un des observables est le changement sensible relativement au temps de parole pris par les stagiaires par rapport au temps laissé aux élèves pour agir et/ou s'exprimer. Alors que durant la deuxième semaine de terrain le temps de parole enseignante avoisinait les $60 \%$, durant les deux tâches de la première leçon de la troisième semaine ce temps a été réduit à $48 \%$ environ. Ce changement quantitatif a été également accompagné de changements qualitatifs : augmentation des gestes de dévolution (consignes plus brèves, davantage de questions adressées aux élèves, régulations moins fréquentes et plus ciblées), ainsi que davantage de temps et d'opportunités laissés aux élèves pour interagir et avec le milieu didactique et dans chaque sous-groupe.

\section{QUELQUES ÉLÉMENTS DE DISCUSSION}

L'utilisation des dispositifs d'autoconfrontation pour la formation constitue un apport dans le cadre de la recherche sur les dispositifs de formation par alternance. Même si le dispositif de recherche présenté n'est qu'à ses débuts, nous avons déjà quelques éléments qui nous incitent à réfléchir sur nos dispositifs de formation professionnelle en termes de "situations didactiques ", dans le sens que leur donne Brousseau (1996). De même que pour les situations scolaires, il est possible de concevoir un milieu didactique en interaction avec lequel les étudiants puissent produire de nouvelles connaissances à partir des incertitudes et des obstacles qui émergent de leur pratique effective. Comme pour les situations scolaires, l'interaction des stagiaires avec et dans le milieu didactique peut être instrumentée par les formateurs universitaires et/ou de terrain dans le but, d'une part, de leur fournir des outils de transformation et d'analyse et, d'autre part, de leur permettre de réguler/modifier activement leurs pratiques.

Le travail sur les séquences EP analysé nous a permis de dégager les potentialités des dispositifs méthodologiques d'autoconfrontation pour la prise de distance analytique, ainsi que comme moyen pour faire émerger l'implicite des pratiques. Les exemples analysés nous ont permis également de montrer la possibilité de surmonter quelques limites et obstacles de ce type de dispositif. En ce sens l'intervention du chercheur dans

8 Nous avons déjà analysé ailleurs (Schubauer \& Rickenmann, REF-2003) quelques problèmes qui se posent aux stagiaires du fait d'être soumis, à la fois, au contrat de formation (posture d'apprenant) et au contrat didactique avec leurs élèves (posture d'enseignant). le choix de structuration des séances vidéoscopées s'avère être un puissant catalyseur des processus potentiellement liés à l'autoconfrontation. Cette intervention rend manifeste la nécessité de ne pas naturaliser les méthodes, notamment dans le cadre des démarches cliniques de la recherche didactique (Leutenegger, 1999).

L'analyse des séquences AP nous a permis d'illustrer cette nécessité d'instrumentation conceptuelle, mobilisée pour l'occasion dans un but formatif. Les concepts sur l'action professorale ont en ce sens une fonction de grille de lecture qui médiatise les lectures de la pratique. Les catégories de l'action professorale ont permis aux étudiants de « voir » une structure plus complexe que la seule organisation par phases que les étudiants ont tendance à produire spontanément (introduction du maitre, tâches de l'élève, bilan ou évaluation). Les fonctions de l'action professorale (définir, dévoluer, réguler, institutionnaliser) leur permettent de valider la structuration initialement proposée en même temps qu'elles incitent à approfondir leur regard sur chaque phase. L'analyse a montré que cette médiation permet aux étudiants de les « re-trouver " à un niveau plus micro, dont ils n'ont souvent pas conscience comme nous l'avons montré avec l'analyse d'autoconfrontation des maîtres dans les séquences EP.

Un deuxième niveau d'instrumentation est basésurl'organisation du travail étudiant en dyades, qui leur permet de capter en même temps deux dimensions fortement liées à la pratique enseignante (posture d'enseignement et posture d'observation). Dans ce cas, la mise à disposition des catégories d'analyse (méso-topo et chrono/génétiques) produit des effets de distanciation et permet d'adopter une conception plus systémique de l'action professorale.

Notons, pour conclure, qu'il ne suffit pas de mettre à disposition des catégories et modèles conceptuels pour aider à la lecture des pratiques, mais qu'il est aussi nécessaire de prévoir des « moments " de formation qui ne soient pas toujours liés aux « urgences " de la pratique. L'autoconfrontation comme partie intégrante d'un milieu didactique de formation (mésogenèse), de même que le temps qui est mis à disposition pour observer, analyser les pratiques et pour modifier les planifications (chronogenèse), constituent des actions de dévolution de la responsabilité associées à une diversification des postures d'apprentissage (topogenèse). 
Séquence du groupe témoin (GT)

1. Organisation en phases et tâches d'apprentissage

\section{Leçon 1}

Activité principale (expérimentale) pour les élèves de 4ème primaire: produire un tableau avec la technique du dripping (20 min.)

Activité secondaire, pour les élèves de 5ème primaire: produire un dessin à main levée de leurs camarades au travail.

Le professeur donne une très brève consigne concernant les règles $\mathrm{du}$ dripping (ne pas toucher la feuille, laissez couler la peinture) (20 secondes). Pour l'autre tâche, il donne pour consigne de dessiner un camarada au travail (10 secondes)

\section{Leçon 2}

Echange autour des productions des élèves (10 min)

Echange assez libre sur les rapports entre les productions d'élèves et quelques reproductions des tableaux de Pollock (20 mn.)

2. Milieu didactique et organisation sociale

Dans la cour de l'école, sur une surface recouverte de plastique protecteur $(20 \mathrm{~m} 2)$, sept élèves produisent individuellement leurs peintures. Ils partagent les pots de peinture situés au centre de la surface. Les choix des couleurs est limité (variable didactique qui ne sera pas mise en évidence lors de la deuxième leçon). La deuxième leçon a eu lieu dans la salle de classe.
Séquence du groupe de stagiaires (GSta)

1. Organización en fases y tareas de aprendizaje

Leçon 1

Première tâche: Observer des reproductions des tableaux de Pollock disposés au centre d'une table autour de laquelle sont situées les élèves; constater qu'elles ne sont pas figuratives; constater qu'elles sont des « traces des gestes de l'artiste »; suggérer le type d'effet que ces tableaux produisent chez celui qui les regarde. (15min.)

Deuxième tâche (tâche principale): produire une peinture " à la manière de Pollock" à partir des thèmes proposés par les élèves comme interprétation des tableaux dans la première tâche. Les thèmes mettent en évidence les effets sur le récepteur plutôt que l'expression. (20 min.)

Dans la consigne, les stagiaires insistent sur la nécessité d'adopter différentes postures corporelles et gestuelles et à utiliser tout l'espace de la feuille (tourner autour de la feuille).

Leçon 2

Première tâche: Echange critique sur les productions des autres groupes. L'objectif est de mettre en évidence des critères qui permettent de valider les productions par rapport au thème et à la fonction que remplissent les éléments techniques (geste et couleurs) dans cette validation.

La consigne insiste sur le fait que la validation doit être basée sur "la manière de peindre" (20 min.)

Deuxième tâche: démonstration des gestes picturaux qui ont été validés durant la première tâche (institutionnalisation) (10 min.)

Troisième tâche: les élèves qui le désirent peuvent donner leur avis sur leur propre expérience esthétique ( $5 \mathrm{~min}$.)

2. Milieu didactique et organisation sociale

Dans la salle de classe, plusieurs surfaces $(4 \mathrm{~m} 2)$ couvertes avec du plastique permettent aux élèves de produire le tableau par groupes de trois élèves. Le groupe doit choisir un thème et le mettre en rapport avec les gestes techniques du dripping, qui n'ont pas été encore nommés, mais qui ont été décrits durant la première tâche (leçon 1). Les élèves de chaque groupe doivent choisir trois pots de peinture parmi quatorze mis à disposition du groupe classe.

Entre la leçon 1 et 2, plusieurs organisations sociales entrent en jeu: travail individuel, groupe-classe, petits groupes. De même, sont sollicités diverses postures d'apprentissage inspirées des pratiques sociales de référence : produire, critiquer, observer, décrire, ... 
Références

Amade-Escot, C., Barrué, J.-P., Bos, J.-C., Duffor, F., Dugrand, M., Terisse, A., (1998). Didactique de l'EPS. Grenoble : La Pensée sauvage.

Amade-Escot, C. (2002). Étude du travail de l'enseignant d'éducation physique dans la classe : contribution des recherches didactiques à l'analyse des pratiques effective. In J.-F. Marcel (Éd).), Les sciences de l'éducation : des recherches, une discipline (pp. 53-78). Paris : L'harmattan, coll. Savoir et formation.

Amade-Escot, C. (2003). La gestion interactive du contrat didactique en volley-ball : agencement des milieux et régulations du professeur (pp. 255-278). In : Didactique de l'éducation physique. (Dir.) Amade-Escot, Paris : Ed. EPS.

Biddle, B.J., Good, T.-L., Goodson, I.F. (eds.) (1997). International Handbook of teachers end teaching (vol 3). Dordrecht: Kluwer.

Bronckart, J.-P. (2005). Vingt-cinq ans de didactique de l'expression écrite. Eléments de bilan et perspectives d'avenir. Revue Suisse des Sciences de l'Education, 27 (3), pp.361-380.

Brousseau, G (1986). Fondements et méthodes de la didactique des mathématiques. Recherches en didactique des mathématiques, La pensée Sauvage, 7(2), 33-115.

Brousseau, G. (1996). L'enseignant dans la théorie des situations didactiques. Actes de la 8 e école d'été de didactique des mathématiques. IREM : Clermont-Ferrand.

Calderhead, J. (1996). Teachers: Beliefs and knowledge. In D.C. Berliner \& R.C. Calfee (Eds.), Handbook of educational psychology (pp. 709-725). New York: Macmillan

Chevallard, Y. (1995). La fonction professorale. Esquisse d'un modèle didactique. In R. Noirfalise (éd.), Actes de la 8ème école d'eté de didactique des mathématiques, p. 83-122.

Chevallard Y., 1999, L'analyse des pratiques enseignantes en théorie anthropologique du didactique. Recherches en Didactique des Mathématiques, 19 (2), 221-265, La Pensée Sauvage, Grenoble.

Chevallard, Y (2004). La place des mathématiques vivantes dans l'éducation secondaire : transposition didactique des mathématiques et nouvelle épistémologie scolaire. 3e Université d'eté Animath, Saint-Flour (Cantal), 22-27 août.

Clot, Y., Faïta, D (2000). Genres et styles en analyse du travail. Concepts et méthodes. Travailler, 4, 7-42.

Crahay, M., Lafontaine, D. (eds) (1989). L'art et la science de l'enseignement. Paris : PUF.

Devolvé, N. (2000). Pour une approche systémique du métier d'enseignant, le point de vue de l'ergonomie. Approches systémiques et recherches en Sciences de l'Education, revue du CREFI, No 3.

Goigoux, R. (2000). Enseigner le lecture à l'école primaire. Dossier d'Habilitation à diriger des recherches, U.F.R. de Sciences de l'éducation, Université de Paris VIII.

Faïta, D. Vieira D. (2003). Réflexions méthodologiques sur l'autoconfrontation croisée. Internet :http://www.scielo.br/scielo.
php?script=sci_arttext\&pid=S0102-44502003000100005

Lave, J., Wenger, E. (1991). Situated Learning : Legitimate peropherical participation. Cambridge: University University Press.

Leutenegger, F. (1999). Contribution à la théorisation d'une clinique pour le didactique. Thèse de Doctorat en sciences de l'éducation. Université de Genève.

Leutenegger, F., Ligozat, F. (2006). Analyse de l'action conjointe professeur-élèves dans le traitement d'un problème d'agrandissement de distances. Séminaire National de DDM..

Leutenegger, F. (2000). Construction d'une « clinique " pour le didactique. Une étude des phénomènes temporelles del'enseignement. Recherches en Didactique des mathématiques, Vol. 20/2, pp. 209-250.

Loquet M., Garnier A. \& Amade-Escot C. (2002) Transmission des savoirs en activités physiques, sportives et artistiques dans des institutions différentes : enseignement scolaire, entraînement sportif, transmission chorégraphique. Revue française de pédagogie, "Vers une didactique comparée ", 141, pp. 99-109.

Mercier, A, Sensevy, G., Schubauer-Leoni, M.-L. (2000). Vers un modèle de l'action didactique du professeur. A propos de la course à vingt. Recherches en didactiques des mathématiques, 20(3), 263-304.

Mili, I., Rickenmann, R. (2005). Approches didactiques dans l'enseignement artistique: une revue de littérature sur la construction de l'expertise culturelle en classe. Revue Suisse des Sciences de l'Education.

Mili, I., Rickenmann, R. (2004). La construction des objets culturels dans l'enseignement artistique et musical. In C. Moro \& R. Rickenmann (éds), Situation éducative et significations (p. 165-196), Bruxelles : DeBoeck.

Portugais, J. (1992). Didactique des mathématiques et formation des enseignants. Berne : Peter Lang.

Revue française de Pédagogie. (2002) Vers une didactique comparée, oct. nov. déc. 141.

Rickenmann, R. (2001). Sémiotique de l'action éducative. In J.-M. Baudouin \& J. Friedrich (éd.), Théories de l'action et éducation (p. 225254), Bruxeles : DeBoeck

Rickenmann, R. (2005). Didactics in art education and appropriation of cultural objects in classroom. A semiotical analysis from a teaching/learning perspective. Communication dans le 1st International Congress of the ISCAR, Sevilla, Spain, Sept 20-24.

Rickenmann, R. (2006a). El rol de los artefactos culturales en la estructuración y gestión de secuencias de enseñanza-aprendizaje. In Conférence invitée, Actes du 1er simposio internacional de educación y formación docente, Universidad de Antioquia, Medellín (Colombia), agosto 29 septiembre 1 ro 2006

Rickenmann, (2006b, sous presse). Metodologías clínicas de investigación en didácticas y formación del profesorado: un estudio de los dispositivos de formación en alternancia, Revista educación, Universidad Nacional de 
Antioquia

Salin, M.H. (1997). Contraintes de la situation didactique et décisions de l’enseignante. In C. Blanchard - Laville (éd.), Variations sur une leçon de mathématiques. Paris : L'Harmattant. P. 31-57.

Sensevy, G (2001). Théories de l'action et action du professeur. In J.-M. Baudouin \& J. Friedrich (éd.), Théories de l'action et éducation (p. 203224), Bruxelles: DeBoeck

Sensevy, G., Mercier, A.., Schubauer-Leoni. M.-L. (2000) Vers un modèle de l'action didactique du professeur à propos de la couse à 20. Recherches en Didactique des Mathématiques, 20 (3) 263-304.

Sensevy, G., Mercier, A. (REF-2003 sous presse) Logiques d'action du professeur et du chercheur. Un essai d'analyse. In Actes du symposium Recherche et intervention en didactiques, Réseau Education Formation (REF), Genève.

Schubauer, R., Rickenmann, R., (REF-2003, sous presse). Un dispositif expérimental de formation aux gestes professionnels. In Actes du symposium Recherche et intervention en didactiques, Réseau Education Formation (REF), Genève.

Schubauer-Leoni, M.-L, (1998). Les sciences didactiques parmi les sciences de l'éducation : l'étude du projet scientifique de la didactique des mathématiques. In R. Hofstetter \& B. Schneuwly (Ed.), Le pari des sciences de l'éducation (Raisons éducatives N¹998, 1-2, pp. 329352). Bruxelles : DeBoeck.

Sieber, P., Thévenaz, T. (2005). Didactique des disciplines et didactique comparée : entre continuité et réorientation. Revue Suisse des Sciences de l'éducation.

Thepaut A. (2003). Echec scolaire et éducation physique et sportive à l'école élémentaire. Etude des interaction maître-élèves dans la construction des savoirs. L'exemple de l'apprentissage de la passe en basket-ball. Thèse de Doctorat. Université de Rennes II, France.

Wirthner, M., Schneuwly B. (2004). Variabilit é et contrainte dans la construction des significations d'un objet d'enseignement. In C. Moro \& R. Rickenmann (Eds), Situation éducative et significations (pp. 107-133), Bruxelles: DeBoeck.

RENÉ RICKENMANN. Doctor en ciencias de la Educación de la Universidad de Ginebra (Suiza). Inicio sus estudios superiores con una licenciatura en la Facultad de Ciencias Sociales de la Universidad Javeriana de Bogotá, y un máster "Sociedad y sistemas de formación" en la Universidad de Ginebra. Director Equipo Semiótica, Educación y Desarrollo (SED). Didáctica de las artes plásticas, Facultad de Sicología y de Ciencias de la Educación, Universidad de Ginebra. Docente en esta última Universidad desde 1995. rene.rickenmann@unige.ch

ADRIÁN CÓRDOBA. Profesor de Educación Física en el programa de formación de profesores de la primaria y miembro del equipo de investigación Semiótica-Educación-Desarrollo (SED) en la facultad de Ciencias de la educación de la Universidad de Ginebra. Diplomado en educación física del Instituto de formación Docente Olavaria (Argentina) y Licenciado en ciencias de la educación de la Universidad de Ginebra (Suiza). Actualmente realiza su trabajo de doctorado en la Facultad de psicología y de ciencias de la educación. Universidad de Ginebra.

Recibido: Agosto 3 de 2008 y aceptado: Septiembre 2 de 2008. adrian.cordoba@unige.ch

Artículo recibido: Marzo de 2009 y aceptado: Julio de 2009. 